\title{
What the Infrared Behavior of QCD Green Functions can tell us about Confinement in the Covariant Gauge ${ }^{a}$
}

\author{
Lorenz von Smekal \\ Universität Erlangen-Nürnberg, Institut für Theoretische Physik III, \\ Staudtstr. 7, 91058 Erlangen, Germany \\ E-mail:smekal@theorie3.physik.uni-erlangen.de \\ Reinhard Alkofer \\ Universität Tübingen, Institut für Theoretische Physik, \\ Auf der Morgenstelle 14, 72076 Tübingen, Germany \\ E-mail: Reinhard.Alkofer@uni-tuebingen.de
}

\begin{abstract}
We review aspects of confinement in the covariant and local description of QCD and discuss to what extend our present knowledge of the infrared behavior of QCD Green functions can support this description. In particular, we emphasize: the positivity violations of transverse gluon and quark states, the Kugo-Ojima confinement criterion, and the conditions necessary to avoid the decomposition property for colored clusters. We summarize how these issues relate to the infrared behavior of the propagators in Landau gauge QCD as extracted from solutions to truncated Dyson-Schwinger equations and lattice simulations.
\end{abstract}

\section{The Covariant Description of Confinement}

Covariant quantum theories of gauge fields require indefinite metric spaces. Abandoning the positivity of the representation space already implies some modifications to the standard (axiomatic) framework of quantum field theory. Maintaining the much stronger principle of locality, great emphasis has been put on the idea of relating confinement to the violation of positivity in QCD. Just as in QED, where the Gupta-Bleuler prescription is to enforce the Lorentz condition on physical states, a semi-definite physical subspace can be defined as the kernel of an operator. The physical states then correspond to equivalence classes of states in this subspace differing by zero norm components. Besides transverse photons covariance implies the existence of longitudinal and scalar photons in QED. The latter two form metric partners in the indefinite space. The Lorentz condition eliminates half of these leaving unpaired states of zero norm which do not contribute to observables. Since the Lorentz condition commutes with the $S$-Matrix, physical states scatter into physical ones exclusively.

Confinement in QCD can be ascribed to an analogous mechanism: Within the framework of BRS algebra, in the simplest version for the BRS-charge $Q_{B}$ and the ghost number $Q_{c}$ (both hermitean with respect to an indefinite inner product) given by,

$$
Q_{B}^{2}=0, \quad\left[i Q_{c}, Q_{B}\right]=Q_{B},
$$

completeness of the nilpotent BRS-charge $Q_{B}$ in a state space $\mathcal{V}$ of indefinite metric is assumed. This charge generates the BRS transformations by ghost number graded commutators $\{$,$\} , i.e.,$

\footnotetext{
${ }^{a}$ Invited talk by L. v. Smekal at "Quark Confinement and the Hadron Spectrum IV", Vienna, July 4-8, 2000.
} 
by commutators or anticommutators for fields with even or odd ghost number, respectively. The semi-definite physical subspace $\mathcal{V}_{\text {phys }}=\operatorname{Ker} Q_{B}$ is defined on the basis of this algebra by those states which are annihilated by the BRS charge $Q_{B}$. Since $Q_{B}^{2}=0$, this subspace contains the space $\operatorname{Im} Q_{B}$ of so-called daughter states which are images of others, their parent states in $\mathcal{V}$. A physical Hilbert space is then obtained as (the completion of) the covariant space of equivalence classes, the BRS-cohomology of states in the kernel modulo those in the image of $Q_{B}$,

$$
\mathcal{H}\left(Q_{B}, \mathcal{V}\right)=\operatorname{Ker} Q_{B} / \operatorname{Im} Q_{B} \simeq \mathcal{V}_{s},
$$

which is isomorphic to the space $\mathcal{V}_{s}$ of BRS singlets. It is easy to see that the image is furthermore contained in the orthogonal complement of the kernel (given completeness they are identical). It follows that states in $\operatorname{Im} Q_{B}$ do not contribute to the inner product in $\mathcal{V}_{\text {phys }}$. Completeness is thereby important in the proof of positivity for physical states 1 because it assures the absence of metric partners of BRS-singlets, so-called "singlet pairs".

With completeness, all states in $\mathcal{V}$ are either BRS singlets in $\mathcal{V}_{s}$ or belong to so-called quartets which are metric-partner pairs of BRS-doublets (of parent with daughter states). This exhausts all possibilities. The generalization of the Gupta-Bleuler condition on physical states, $Q_{B}|\psi\rangle=0$ in $\mathcal{V}_{\text {phys }}$, eliminates half of these metric partners leaving unpaired states of zero norm which do not contribute to any observable. This essentially is the quartet mechanism: Just as in QED, one such quartet, the elementary quartet, is formed by the massless asymptotic states of longitudinal and timelike gluons together with ghosts and antighosts which are thus all unobservable. In contrast to QED, however, one expects the quartet mechanism also to apply to transverse gluon and quark states, as far as they exist asymptotically. A violation of positivity for such states then entails that they have to be unobservable also. The combined evidence for this collected below provides strong indication in favor of such a violation for possible transverse gluon states.

The members of quartets are frequently said to be confined kinematically. This is no comprehensive explanation of confinement but it is one aspect in its present description. In particular, asymptotic transverse gluon and quark states may or may not exist in the indefinite metric space $\mathcal{V}$. If either of them do exist and the Kugo-Ojima criterion is realized (see below), they belong to unobservable quartets. In that case, the BRS-transformations of their asymptotic fields entail that they form these quartets together with ghost-gluon and/or ghost-quark bound states, respectivelye It is furthermore crucial for confinement, however, to have a mass gap in transverse gluon correlations, i.e., the massless transverse gluon states of perturbation theory have to dissappear even though they should belong to quartets due to color antiscreening and superconvergence in QCD for less than 10 quark flavors 3.4

The quantum mechanical interpretation in terms of transition probabilities and measurements holds between physical states and for expectation values in $\mathcal{V}_{\text {phys }}$ of those operators for which all contributions from zero norm states vanish. For a (smeared local) operator $A$ to be observable in this sense it is necessary to be BRS-closed, $\left\{i Q_{B}, A\right\}=0$, which coincides with the requirement of its local gauge invariance. It then follows that for all states generated from the vacuum $|\Omega\rangle$ by any such observable one has $\left\langle\Omega\left|A^{\dagger} A\right| \Omega\right\rangle \geq 0$.

If this construction is shown to apply to a QCD description of hadrons as the genuine physical particles of $\mathcal{H}$, one concludes from physical $S$-matrix unitarity (with respect to the indefinite inner product) that absorptive thresholds in hadronic amplitudes can only be due to intermediate hadronic states. The $S$-matrix commutes with the BRS-charge, i.e., it is an observable in the above sense which transforms physical states into physical ones exclusively 
and without measurable effects from possible zero norm components 3 One shows likewise in this description that anomalous thresholds arise only from the substructure of a given hadron as being composed of other hadrons. The argument to establish this employs standard analyticity properties for hadronic amplitudes and crossing to relate them to absorptive singularitips of other hadronic amplitudes which can in turn be due to intermediate hadronic states only

Confinement depends on the realization of the unfixed global gauge symmetries in this formulation. The identification of the BRS-singlets in the physical Hilbert space $\mathcal{H}$ with color singlets is possible only if the charge of global gauge transformations is BRS-exact and unbroken, i.e., well-defined in the whole of the indefinite metric space $\mathcal{V}$. The sufficent conditions for this are provided by the Kugo-Ojima criterion: Considering the globally conserved current

$$
J_{\mu}^{a}=\partial_{\nu} F_{\mu \nu}^{a}+\left\{Q_{B}, D_{\mu}^{a b} \bar{c}^{b}\right\} \quad\left(\text { with } \partial_{\mu} J_{\mu}^{a}=0\right),
$$

one realizes that the first of its two terms corresponds to a coboundary with respect to the spacetime exterior derivative while the second term is a BRS-coboundary with charges denoted by $G^{a}$ and $N^{a}$, respectively,

$$
Q^{a}=\int d^{3} x \partial_{i} F_{0 i}^{a}+\int d^{3} x\left\{Q_{B}, D_{0}^{a b} \bar{c}^{b}\right\}=G^{a}+N^{a} .
$$

For the first term herein there are only two options, it is either ill-defined due to massless states in the spectrum of $\partial_{\nu} F_{\mu \nu}^{a}$, or else it vanishes.

In QED massless photon states contribute to the analogues of both currents in (3), and both charges on the r.h.s. in (4) are separately ill-defined. One can employ an arbitrariness in the definition of the generator of the global gauge transformations (4), however, to multiply the first term by a suitable constant so chosen that these massless contributions cancel. This way one obtains a well-defined and unbroken global gauge charge which replaces the naive definition in (何) above Roughly speaking, there are two independent structures in the globally conserved gauge currents in QED which both contain massless photon contributions. These can be combined to yield one well-defined charge as the generator of global gauge transformations leaving any other combination spontaneously broken, such as the displacement symmetry which lead to the identification the photon with its massless Goldstone bosone 2 |

If $\partial_{\nu} F_{\mu \nu}^{a}$ contains no massless discrete spectrum on the other hand, i.e., if there is no massless particle pole in the Fourier transform of transverse gluon correlations, then $G^{a} \equiv 0$. In particular, this is the case for channels containing massive vector fields in theories with Higgs mechanism, and it is expected to be also the case in any color channel for QCD with confinement for which it actually represents one of the two conditions formulated by Kugo and Ojima. In both these situations one first has equally, however,

$$
Q^{a}=N^{a}=\left\{Q_{B}, \int d^{3} x D_{0}^{a b} \bar{c}^{b}\right\}
$$

which is BRS-exact. The second of the two conditions for confinement is that this charge be well-defined in the whole of the indefinite metric space $\mathcal{V}$. Together these conditions are sufficient to establish that all BRS-singlet physical states in $\mathcal{H}$ are also color singlets, and that all colored states are thus subject to the quartet mechanism. The second condition thereby provides the essential difference between Higgs mechanism and confinement. The operator $D_{\mu}^{a b} \bar{c}^{b}$ determining the charge $N^{a}$ will in general contain a massless contribution from the 
elementary quartet due to the asymptotic field $\bar{\gamma}^{a}(x)$ in the antighost field, $\bar{c}^{a} \stackrel{x_{0} \rightarrow \pm \infty}{\longrightarrow} \bar{\gamma}^{a}+\cdots$ (in the weak asymptotic limit),

$$
D_{\mu}^{a b} \bar{c}^{b} \stackrel{x_{0} \rightarrow \pm \infty}{\longrightarrow}\left(\delta^{a b}+u^{a b}\right) \partial_{\mu} \bar{\gamma}^{b}(x)+\cdots .
$$

Here, the dynamical parameters $u^{a b}$ determine the contribution of the massless asymptotic state to the composite field $g f^{a b c} A_{\mu}^{c} \bar{c}^{b} \stackrel{x_{0} \rightarrow \pm \infty}{\longrightarrow} u^{a b} \partial_{\mu} \bar{\gamma}^{b}+\cdots$. These parameters can be obtained in the limit $p^{2} \rightarrow 0$ from the Euclidean correlation functions of this composite field, e.g.,

$$
\int d^{4} x e^{i p(x-y)}\left\langle D_{\mu}^{a e} c^{e}(x) g f^{b c d} A_{\nu}^{d}(y) \bar{c}^{c}(y)\right\rangle=:\left(\delta_{\mu \nu}-\frac{p_{\mu} p_{\nu}}{p^{2}}\right) u^{a b}\left(p^{2}\right) .
$$

The theorem by Kugo and Ojima asserts that all $Q^{a}=N^{a}$ are well-defined in the whole of $\mathcal{V}$ (and do not suffer from spontaneous breakdown), if and only if

$$
u^{a b} \equiv u^{a b}(0) \stackrel{!}{=}-\delta^{a b} .
$$

Then the massless states from the elementary quartet do not contribute to the spectrum of the current in $\mathrm{N}^{a}$ and the equivalence between physical BRS-singlet states and color singlets is established国国

In contrast, if $\operatorname{det}(\mathbb{1}+u) \neq 0$, the global gauge symmetry generated by the charges $Q^{a}$ in eq. (1) is spontaneuosly broken in each channel in which the gauge potential contains an asymptotic massive vector field 1 . While this massive vector state results to be a BRS-singlet, the massless Goldstone boson states which usually occur in some components of the Higgs field, replace the third component of the vector field in the elementary quartet and are thus unphysical. Since the broken charges are BRS-exact, this hidden symmetry breaking is not directly observable in the Hilbert space of physical states $\mathcal{H}$.

It is nevertheless instructive to classify the different szenarios according to the realization of the global gauge symmetry on the whole of the indefinite metric space $\mathcal{V}$ of covariant gauge theories. If it is unbroken, i.e., as for QED and QCD, the first condition is crucial for confinement. Namely, it is then necessary to have a mass gap in transverse gluon correlations (i.e., in $\partial_{\nu} F_{\mu \nu}^{a}$ ), since otherwise one could in principle have non-local physical (BRS-singlet and thus gauge invariant) states which are no color singlets, just as one has non-local gauge invariant charged states in QED (e.g., the state of one electron alone in the world with its long-range Coulomb tail). Indeed, with unbroken global gauge invariance QED and QCD have in common that any gauge invariant localized state must be chargeless/colorless 2 The question is the extension to non-local states as approximated by local ones. In QED this leads to the so-called charge superselection sectors 1 and non-local physical states with charge arise. If in QCD, with unbroken global gauge symmetry and mass gap, every gauge-invariant state can be approximated by gauge-invariant localized ones (which are colorless), one concludes that every gauge invariant (BRS-singlet) state in $\mathcal{H}$ must be a color singlet.

The (2nd condition in the) Kugo-Ojima confinement criterion, $u=-\mathbb{1}$ leading to welldefined charges $N^{a}$, can in Landau gauge be shown by standard arguments employing DysonSchwinger equatigns and Slavnov-Taylor identities to be equivalent to an infrared enhanced ghost propagator In momentum space the nonperturbative ghost propagator of Landau gauge QCD is related to the form factor occuring in the correlations of eq. (7) as follows,

$$
D_{G}(p)=\frac{-1}{p^{2}} \frac{1}{1+u\left(p^{2}\right)}, \text { with } u^{a b}\left(p^{2}\right)=\delta^{a b} u\left(p^{2}\right) .
$$


The Kugo-Ojima criterion, $u(0)=-1$, thus entails that the Landau gauge ghost propagator should be more singular than a massless particle pole in the infrared. Indeed, we will present quite compelling evidence for this exact infrared enhancement of ghosts in Landau gauge.

The remaining dynamical aspect of confinement in this formulation resides in the cluster decomposition property of local quantum field theory 9 Including the indefinite metric spaces of covariant gauge theories it can roughly be summarized as follows: For the vacuum expectation values of two (smeared local) operators $A$ and $B$, translated to a large spacelike separaration $R$ of each other one obtains the following bounds depending on the existence of a finite gap $M$ in the spectrum of the mass operator in $\mathcal{V}^{\mathrm{B}}$

$$
\begin{aligned}
\mid\langle\Omega|A(x) B(0)| \Omega\rangle & -\langle\Omega|A(x)| \Omega\rangle\langle\Omega|B(0)| \Omega\rangle \mid \\
& \leq \begin{cases}\text { Const. } \times R^{-3 / 2+2 N} e^{-M R}, & \text { mass gap } M, \\
\text { Const. } \times R^{-2+2 N}, & \text { no mass gap },\end{cases}
\end{aligned}
$$

for $R^{2}=-x^{2} \rightarrow \infty$. Herein, positivity entails that $N=0$, but a positive integer $N$ is possible for the indefinite inner product structure in $\mathcal{V}$. Therefore, in order to avoid the decomposition property for products of unobservable operators $A$ and $B$ which together with the Kugo-Ojima criterion is equivalent to avoiding the decomposition property for colored clusters, there should be no mass gap in the indefinite space $\mathcal{V}$. Of course, this implies nothing on the physical spectrum of the mass operator in $\mathcal{H}$ which certainly should have a mass gap. In fact, if the cluster decomposition property holds for a product $A(x) B(0)$ forming a (smeared local) observable, it can be shown that both $A$ and $B$ are observables themselves. This then eliminates the possibil-

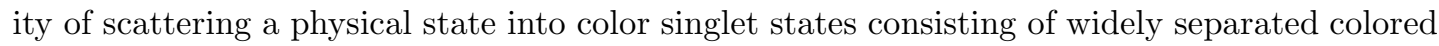
clusters (the "behind-the-moon" problem)

The necessity for the absence of the massless particle pole in $\partial_{\nu} F_{\mu \nu}^{a}$ in the Kugo-Ojima criterion shows that the (unphysical) massless correlations to avoid the cluser decomposition property are not the transverse gluon correlations. An infrared suppressed propagator for the transverse gluons in Landau gauge confirms this condition. This holds equally well for the infrared vanishing propagator obtained from DSEs 10 and conjectured in the studies of the implications of the Gribov horizon 11212 as for the infrared suppressed but possibly finite ones extraced from improved lattice actions for quite large volumes 13

\section{The Infrared Behavior of Gluon and Ghost Propagators}

In Landau gauge the two invariant functions $Z\left(k^{2}\right)$ and $G\left(k^{2}\right)$ in (Euclidean) momentum space parametrize the structure of the gluon and ghost propagators, respectively, as follows (with $G\left(k^{2}\right)=1 /\left(1+u\left(k^{2}\right)\right)$, c.f., eq. (9)),

$$
D_{\mu \nu}(k)=\frac{Z\left(k^{2}\right)}{k^{2}}\left(\delta_{\mu \nu}-\frac{k_{\mu} k_{\nu}}{k^{2}}\right), \quad D_{G}(k)=-\frac{G\left(k^{2}\right)}{k^{2}} .
$$

One approach that proved quite useful to study the non-perturbative infrared behavior of these functions is provided by solutions to truncated Dyson-Schwinger equations(DSEs) for the propagators, i.e., their equations of motion 514 The known structures in the 3-point vertex functions, most importantly from their Slavnov-Taylor identities and exchange symmtries, are thereby employed to establish closed systems of non-linear integral equations that are complete on the level of the gluon, ghost and quark propagators in Landau gauge QCD. This is possible with 


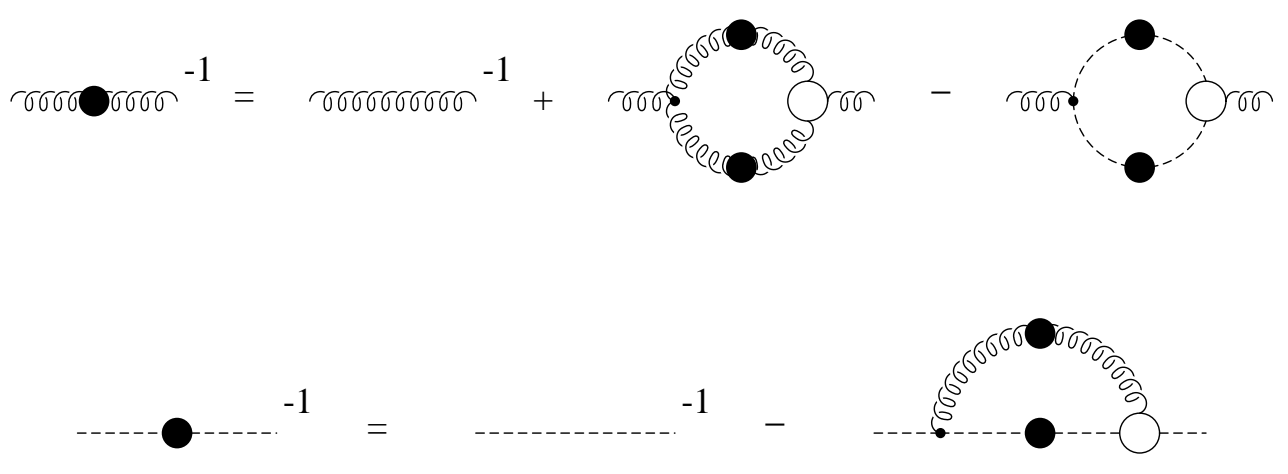

Figure 1: Diagrammatic representation of the truncated system of gluon and ghost DSEs.

systematically neglecting contributions from explicit 4-point vertices to the propagator DSE as well as non-trivial 4-point scattering kernels in the constructions of the 3-point vertices 10 For the pure gauge theory this leads to the propagators DSEs diagrammatically represented in Fig. 11 with the 3-gluon and ghost-gluon vertices (the open circles) expressed in terms of the two functions $Z$ and $G$. Emplpying a one-dimensional approximation one obtains the numerical solutions sketched in Fig. 2010 . 15

Asymptotic expansions of the solutions in the infrared are known analytically. The leading infrared behavior is thereby uniquely determined by one exponent $\kappa=(61-\sqrt{1897}) / 19 \approx 0.92$,

$$
Z\left(k^{2}\right) \stackrel{k^{2} \rightarrow 0}{\sim}\left(\frac{k^{2}}{\sigma}\right)^{2 \kappa} \text { and } G\left(k^{2}\right) \stackrel{k^{2} \rightarrow 0}{\sim}\left(\frac{\sigma}{k^{2}}\right)^{\kappa}
$$

for which the bounds $0<\kappa<1$ can be established 10 The renormalization group invariant momentum scale $\sigma$ represents the free parameter at this point. Its relation to the scale $\Lambda$ of perturbative QCD is rather indirect, but a rough estimate of its value may be obtained from the corresponding running coupling shown in Fig. 3 (see below). From $\alpha_{S}(\mu)=0.118$ at $\mu=M_{Z}=91.2 \mathrm{GeV}$, the mass of the $Z$-boson, one then obtains $\sigma \cong(350 \mathrm{MeV})^{2}$ which, as a qualitative test, with $M_{Z} / M_{\tau} \cong 51.5$ yields $\alpha_{S}\left(M_{\tau}\right)=0.38$ at the $\tau$-mass 10

The infrared behavior in eqs. (12) was later confirmed qualitatively by studies of further truncated DSEs 16 Neither does it thus seem to depend on the particular 3-point vertices nor on the one-dimensional approximation employed in the original solutions. While the gluon propagator is found to vanish for small spacelike momenta in this way, an apparent contradiction with earlier DSE studies that implied its infrared enhancement7 can be understood from the observation that the previously neglected ghost propagator now assumes just this: An infrared enhancement of ghosts corresponding to $u(0)=-1$ which alongside with the absence of massless asymptotic transverse gluon states for $Z(0)=0$ is predicted by the Kugo-Ojima confinement criteriong

There are also recent lattice simulations which test this criterion directly 18 Instead of $u^{a b}=-\delta^{a b}$ they obtain numerical values of around $u=-0.7$ for the unrenormalized diagonal parts and zero (within statistical errors) for the off-diagonal parts. After renormalization, diagonal parts very close to -1 result. Taking into account the finite size effects on the lattices employed in the simulations, these preliminary results might be perfectly reconciled with the 


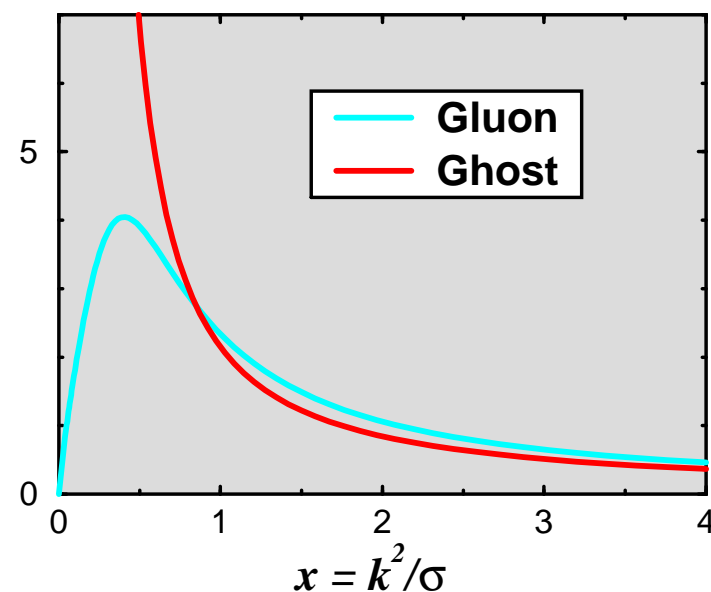

Fig. 2: DSE solutions for $Z(x)$ and $G(x)\lfloor$

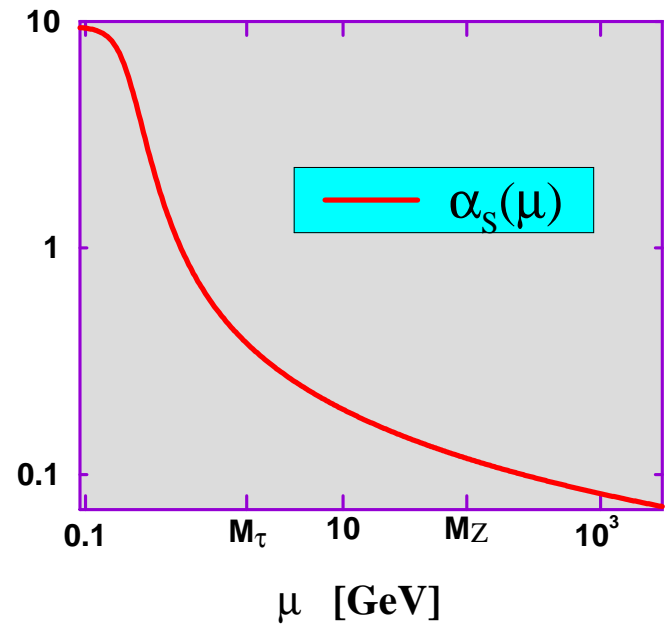

Fig. 3: $\alpha_{S}$ from the solutions in Fig. 2.

Kugo-Ojima confinement criterion (8).

Positivity violations of transverse gluon states are manifest in the spectral representation of (the relevant part of) the gluon propagator,

$$
D\left(p^{2}\right):=\frac{Z\left(p^{2}\right)}{p^{2}}=\int_{0}^{\infty} d m^{2} \frac{\rho\left(m^{2}\right)}{p^{2}+m^{2}} .
$$

From color antiscreening and unbroken global gauge summetry in QCD the spectral density herein asymptotically is negative and superconvergent

$$
\rho\left(k^{2}\right) \stackrel{k^{2} \rightarrow \infty}{\sim}-\frac{\gamma g^{2}}{k^{2}}\left(g^{2} \ln \frac{k^{2}}{\Lambda^{2}}\right)^{-\gamma-1}, \text { and } \int_{0}^{\infty} d m^{2} \rho\left(m^{2}\right)=\left(\frac{g_{0}^{2}}{g^{2}}\right)^{\gamma} \rightarrow 0,
$$

since $\gamma>0$ for $N_{f} \leq 9$ in Landau gauge. This imlies that it contains contributions from quartet states (and does therefore not need to be gauge invariant unlike in QED). Here, we consider the one-dimensional Fourier transform

$$
D\left(t, \mathbf{p}^{2}\right)=\int \frac{d p_{0}}{2 \pi} \frac{Z\left(p_{0}^{2}+\mathbf{p}^{2}\right)}{p_{0}^{2}+\mathbf{p}^{2}} e^{i p_{0} t}=\int_{\sqrt{\mathbf{p}^{2}}}^{\infty} d \omega \rho\left(\omega^{2}-\mathbf{p}^{2}\right) e^{-\omega t},
$$

which for $\rho \geq 0$ had to be positive definite (and one had $\frac{d^{2}}{d t^{2}} \ln D(t, \mathbf{p}) \geq 0$ ). This is clearly not the case for the DSE solution shown in Fig. A which violates reflection positivity 10 Even though no negative $D\left(t, \mathbf{p}^{2}\right)$ have been reported in lattice calculations yet, the available results 19 e.g., see Fig. 同, agree in indicating that $\ln D\left(t, \mathbf{p}^{2}\right)$ is not the convex function of the Euclidean time it should be for positive $\rho 22,21$ These are non-perturbative verifications of the positivity violation for transverse gluon states which already occur in perturbation theory. More significant for confinement is the fact that no massless single transverse gluon contribution to $\rho$ exists for $Z(0)=0$.

${ }^{b}$ This expresses the fact that any 2-point function is analytic in the cut complex $p^{2}$-plane with singularities along the time-like real axis only which holds due to the spectrum condition in the local description also with indefinite metric. 


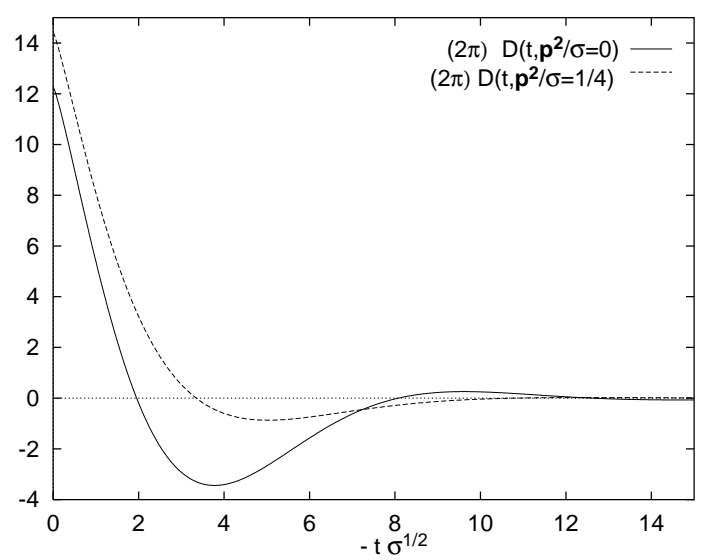

Fig. 4: $D\left(t, \mathbf{p}^{2}\right)$ from DSEs for the gluon renormalization function $Z$ in Fig. 2 .

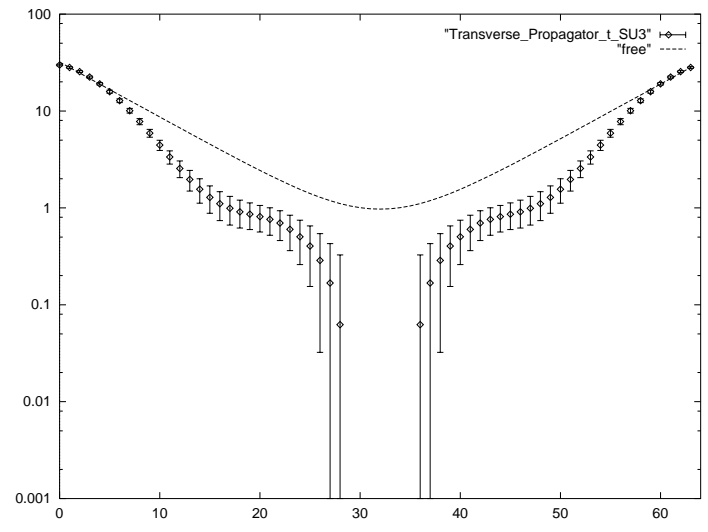

Fig. 5: $D(t, \mathbf{p})$ at $(a \mathbf{p})=(2 \pi / 48,0,0)$ for $\beta=6.8$ on a $48^{3} \times 64$ lattice and the free periodic one $\propto \cosh \left(2 \pi\left(t-N_{t} / 2\right) / 48\right) 21$

In Landau gauge, the non-renormalization of the slyon-ghost vertex offers a convenient possibility to define a nonperturbative running coupling 6 An infrared fixed point, as obtained from the DSE solutions for this coupling shown in Fig. [3,

$$
\alpha_{S}(\mu)=\frac{g^{2}}{4 \pi \beta_{0}} Z\left(\mu^{2}\right) G^{2}\left(\mu^{2}\right) \stackrel{\mu \rightarrow 0}{\longrightarrow} \frac{16 \pi}{3 N_{c}}\left(\frac{1}{\kappa}-\frac{1}{2}\right)^{-1} \simeq 9.5,
$$

which determines the 2-point interactions of color-octet quark currents, thereby implied the existence of the unphysical massless states necessary to escape the cluster decomposition of colored clusters. The corresponding massless single particle poles should occur in colored composite operators and by virtue of the Kugo-Ojima criterion belong to unobservable quartets. An independent verification of this result would thus be desirable. Some implications towards an infrared finite running coupling as analogously being extracted from the 3-gluon vertex might be seen in lattice calculations 23 A potential problem thereby arises in presence of the infrared enhanced ghost propagator as entailed by the Kugo-Ojima friterion for the Landau gauge, if asymmetric momentum subtraction schemes are employed 122 An ideal comparison would therefore be possible from a lattice study of the ghost-gluon vertex in a symmetric momentum subtraction scheme 18

Confirmation of the important result that the gluon renormalization function vanishes in the infrared and no massless asymptotic transverse gluon states ocfyr, i.e., $Z(0)=0$, is seen in Fig. 6, where the DSE solution of Fig. 22 is compared to lattice data 24 and it was further verified recently with improved lattice actions for large volumes 13 This infrared suppression as seen in lattice calculations thereby seems to be weaker than the DSE result, apparently giving rise to an infrared finite gluon propagator $D(k) \sim Z\left(k^{2}\right) / k^{2}$ (corresponding to an exponent $\kappa=1 / 2$ in (12)), but a vanishing one does not seem to be ruled out for the infinite volume limiter Similar results with finite $D(0)$ are also reported from the Laplacian gauge which practically avoids Gribov copies 25

The infrared enhanced DSE solution for ghost propagator is compared to lattice data in Fig. 7. One observes quite compelling agreement, the numerical DSE solution fits the lattice data at low momenta $(x \leq 1)$ significantly better than the fit to an infrared singular form with integer exponents, $D_{G}\left(k^{2}\right)=c / k^{2}+d / k^{4}$. Though low momenta $(x<2)$ were excluded in 


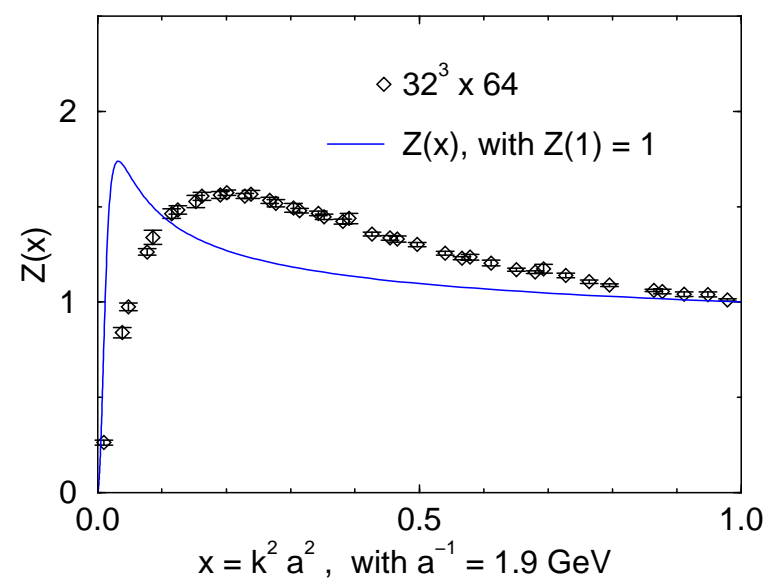

Fig. 6: The gluon renormalizqtion function from the DSE solutions of reflo (solid line) and from the lattice data of refes.

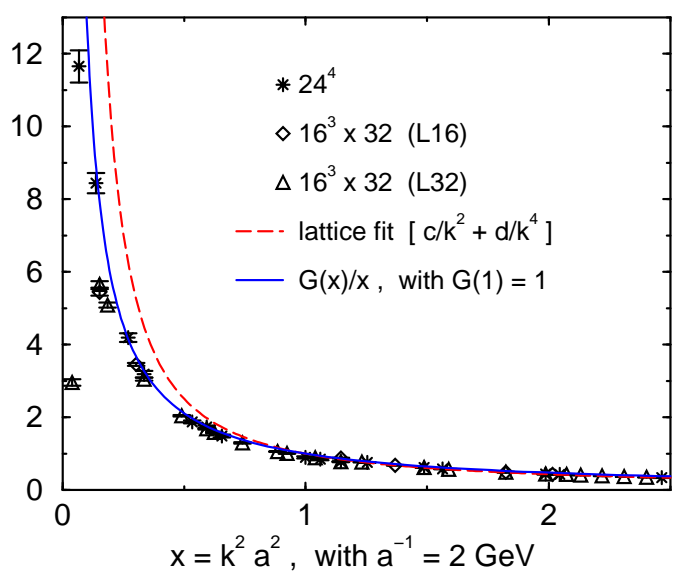

Fig. 7: The ghost propagator from DSEs in ref 10 (solid line) compared to data and fit (dashed with $c a^{2}=0.744, d a^{4}=0.256$ for $x \geq 2$ ) from ref 26 .

this fit, the authors concluded that no reasonable fit of such a form was otherwise possible 26 Therefore, apart from the question about a possible maximum at the very lowest momenta, the lattice calculation seems to confirm the infrared enhanced ghost propagator with a non-integer exponent $0<\kappa<1$. The same qualitativesonclusion has in fact been obtained in a lattice calculation of the ghost propagator in $S U(2) 27$ where its infrared dominant part was fitted best by $D_{G} \sim 1 /\left(k^{2}\right)^{1+\kappa}$ for an exponent $\kappa$ of roughly 0.3 (for $\beta=2.7$ ).

To summarize, the qualitative infrared behavior in eqs. (12), an infrared suppression of the gluon propagator together with an infrared enhanced ghost propagator as predicted by the Kugo-Ojima criterion for the Landau gauge, is confirmed by the presently availabe lattice calculations. The exponents obtained therein $(0<\kappa \leq 0.5)$ both seem to be consistently smaller than the one obtained in solving their DSEs. Whether the lattice data for the infrared behavior of both propagators can thereby also be determined from one unique exponent $0<\kappa<1$, has not yet been investigated to our knowledge.

L.v.S. thanks the organizers for this stimulating conference and for the hospitality extended to him during his stay at Vienna. This work was supported in part by the DFG (Al 279/3-3).

1. T. Kugo and I. Ojima, Prog. Theor. Phys. Supl. 66 (1979) 1.

2. N. Nakanishi and I. Ojima, Covariant Operator Formalism of Gauge Theories and Quantum Gravity, World Scientific Lecture Notes in Physics, Vol. 27, 1990; and references therein.

3. R. Oehme and W. Zimmermann, Phys. Rev. D21 (1980) 471; ibid., 1661;

R. Oehme, Phys. Rev. D42 (1990) 4209, Phys. Lett. B252 (1990) 641.

4. K. Nishijima, Int. J. Mod. Phys. A9 (1994) 3799, ibid., A10 (1995) 3155; see also, Czech. J. Phys. 46 (1996) 1.

5. R. Alkofer and L. v. Smekal, The Infrared Behavior of QCD Green's Functions, submitted to Phys. Rep., hep-ph/0007355; and references therein.

6. R. Oehme, Int. J. Mod. Phys. A10 (1995) 1995. 
7. T. Kugo, at Int. Symp. BRS Sym., Kyoto, Sep. 1995, hep-th/9511033.

8. F. Lenz et al., Ann. Phys. 233 (1994) 17; ibid., 51.

9. R. Haag, Local Quantum Physics, Springer, 1996; and references therein.

10. L. v. Smekal, A. Hauck and R. Alkofer, Phys. Rev. Lett. 79 (1997) 3591; Ann. Phys. 267 (1998) 1.

11. V. N. Gribov, Nucl. Phys. B139 (1978) 1.

12. D. Zwanziger, Nucl. Phys. B378 (1992) 525; ibid., B412 (1994) 657.

13. F. Bonnet et al., hep-lat/0002020; see A. G. Williams, these proceedings.

14. For applications of DSEs to QCD at finite temperature and density, see C. D. Roberts and S. M. Schmidt, Prog. Part. Nucl. Phys. 45 (2000).

15. A. Hauck, L. v. Smekal and R. Alkofer, Comput. Phys. Commun. 112 (1998) 166.

16. D. Atkinson and J. C. R. Bloch, Mod. Phys. Lett. A13 (1998) 1055; Phys. Rev. D58 (1998) 094036.

17. R. Alkofer et al., in Quark Confinement and the Hadron Spectrum II, World Scientific (1997), 258, hep-ph/9608471: A. Hauck, L. v. Smekal and R. Alkofer, Comput. Phys. Commun. 112 (1998) 149; and references therein.

18. H. Nakajima and S. Furui, Nucl. Phys. B (Proc. Suppl.) 83-84 (2000) 521; hep-lat/0006002; see S. Furui, these proceedings.

19. J. E. Mandula, Phys. Rep. 315 (1999) 273; and references therein.

20. J. E. Mandula and M. Ogilvie, Phys. Lett. B185 (1987) 127.

21. A. Nakamura et al., in RCNP Confinement 1995, hep-lat/9506024;

H. Aiso et al., Nucl. Phys. (Proc. Suppl.) B53 (1997) 570.

22. R. Alkofer, S. Ahlig and L. v. Smekal, Fizika B8 (1999), 277; in Understanding Deconfinement in $Q C D$, World Scientific (1999), 196, hep-ph/9905324; R. Alkofer and

L. v. Smekal, hep-ph/0004141.

23. B. Allés et al., Nucl. Phys. B502 (1997) 325;

P. Boucaud et al., JHEP 10 (1998) 017; ibid. 12 (1998) 004.

24. D. Leinweber, J. I. Skullerud, and A. G. Williams, Phys. Rev. D58 (1998) 031501.

25. C. Alexandrou, Ph. de Forcrand and E. Follana, hep-lat/0008012; hep-lat/0009003; see C. Alexandrou, these proceedings.

26. H. Suman and K. Schilling, Phys. Lett. B373 (1996) 314.

27. A. Cucchieri, Nucl. Phys. B508 (1997) 353; in Understanding Deconfinement in QCD, World Scientific (1999), hep-lat/9908050. 